\title{
IMAGING OF FAST MOVING TARGETS USING UNDERSAMPLED SAR RAW-DATA*
}

\author{
Paulo A. C. Marques \\ ISEL-DEEC \\ R. Conselheiro Emídio Navarro 1, \\ 1949-014 Lisboa Portugal \\ E-mail: pmarques@isel.pt
}

\begin{abstract}
In this paper we propose a novel methodology to estimate the true velocity of fast moving targets using a single synthetic aperture radar sensor without increasing the Pulse Repetition Frequency. The basic reasoning is that, although the returned echoes may be aliased in the azimuth direction, its phase is informative with respect to the moving target trajectory parameters. We will use this knowledge to retrieve the necessary information to achieve focused images of fast moving targets on their correct azimuth positions.
\end{abstract}

\section{INTRODUCTION}

Figure 1 shows a typical Synthetic Aperture Radar (SAR) scenario. As the radar travels at constant velocity along the flight path (azimuth direction), short microwave pulses are transmitted at regular intervals and the corresponding echoes are recorded. High resolution in azimuth is achieved by synthesizing a large aperture, exploiting the relative motion between the platform and the ground. The ground is composed of static scatterers with reflectivity $f_{n}$, and a moving target with reflectivity $f_{m}$. If the moving target signature is processed as if it was a static one, the resulting SAR image shows it defocused and/or at wrong positions, depending on the motion direction [1], [2, ch. 6.7]. A moving target in the azimuth direction appears blurred whereas a moving target in the range direction appears misplaced. To obtain focused and correctly positioned images of moving objects it is necessary to know the moving target velocity vector. Methods to retrieve the full velocity vector of a moving target using more than one receiving antenna have recently been published. Examples are the multi-channel SAR [3], the linear antenna array or velocity SAR (VSAR) [4], the multi-frequency antenna array SAR (MF-SAR)[5].

In the recent works [6] and [7] these authors have shown that it is possible to infer the complete velocity vector of a

${ }^{*}$ THIS WORK WAS SUPPORTED BY THE FUNDAÇÃO PARA A CIÊNCIA E TECNOLOGIA, UNDER THE PROJECT POSI/34071/CPS/2000.
José M. B. Dias

\author{
IST-IT \\ Torre Norte, Piso 10, Av. Rovisco Pais, \\ 1049-001 Lisboa Portugal \\ E-mail: bioucas@1x.it.pt
}

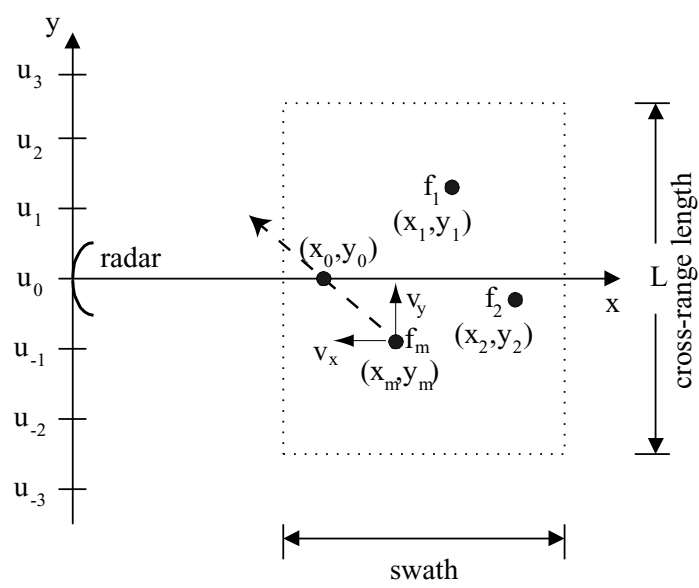

Fig. 1. Considered SAR geometry.

moving target, using SAR data from a single sensor. Basically, we have exploited the structure of the returned echo of a point-like moving target: in the slow-time frequency domain, it is a scaled and shifted replica of the antenna radiation pattern, immersed in noise. An optimal estimator for the full velocity vector was therein presented. As in other proposed methodologies to estimate the speed of moving targets, the maximum range speed that is possible to infer without ambiguity, using a single sensor and a uniform pulse scheduling, is proportional to the used Pulse Repetition Frequency (PRF) [8]. If the signal is aliased (the induced Doppler shift exceeds $P R F / 2$ ), it has been accepted that the true moving targets velocity cannot be uniquely determined. To resolve such targets, the obvious solution consists in increasing the PRF [8]. An alternative solution using a non-uniform PRF was proposed in [9]. PRF increasing leads both to an increase in the memory requirements to store the received signal and to a decrease of the maximum unambiguous range swath. The use of a non-uniform PRF needs a non-conventional pulse scheduling. Moreover, non-uniform sampling introduces complexity in image reconstruction algorithms. 
In this paper we propose a novel methodology to estimate the true velocity of fast moving targets using a single antenna without increasing the PRF. The basic reasoning is that, although the signal may be aliased in the azimuth direction, its phase is informative with respect to the moving target trajectory parameters. We will use this knowledge to retrieve the necessary information to achieve focused images of fast moving targets.

\section{PROPOSED APPROACH}

Consider a moving target with slant-range and cross-range coordinates $\left(x_{m}, y_{m}\right)$ when the platform is at position $y=$ 0 , and speed vector $\left(v_{x}, v_{y}\right)=\left(\mu_{m} v_{r}, b_{m} v_{r}\right)$ defined in the slant-plane $(x, y)$; symbol $v_{r}$ denotes the platform speed and $\left(\mu_{m}, b_{m}\right)$ is the target relative velocity with respect to the radar. When the SAR platform is broadside to the moving target it is positioned at coordinates $\left(x_{0}, y_{0}=u_{0}\right)$. When the radar is positioned at coordinate $y=u^{\prime}$, the corresponding received echo from a moving target, after quadrature demodulation and pulse compression, is

$$
s\left(u^{\prime}, x\right)=a\left(y_{m}-\nu u^{\prime}\right) p_{c}\left(x-r\left(u^{\prime}\right)\right) f_{m} \exp ^{-j 2 k_{0} r\left(u^{\prime}\right)},
$$

where $a(u)$ is the antenna radiation pattern (it is assumed that the dependency of $a(u, x)$ on $x$ is negligible and that there are no pointing errors of the antenna $\left.{ }^{1}\right)$; function $p_{c}(x)$ denotes the compressed emitted pulse in the range direction, $k_{0} \equiv 2 \pi / \lambda_{0}$ is the wavenumber at wavelength $\lambda_{0}$, and $r\left(u^{\prime}\right)$ is the distance between the platform and the moving target, given by

$$
r\left(u^{\prime}\right)=\sqrt{\left(x_{m}-\mu_{m} u^{\prime}\right)^{2}+\left(y_{m}-\nu_{m} u^{\prime}\right)^{2}},
$$

where $\nu_{m} \equiv 1+b_{m}$. Defining $u \equiv u^{\prime}-u_{0}, r\left(u^{\prime}\right)$ can be written as

$$
r(u)=\sqrt{\left(x_{0}-\mu_{m} u\right)^{2}+\left(y_{0}-\nu_{m} u\right)^{2}} .
$$

Approximating $r(u)$ by a series expansion about $u=0$, and retaining only the terms through the quadratic, results

$$
r(u) \approx r_{0}-\underbrace{\frac{x_{0} \mu_{m}+\nu_{m} y_{0}}{r_{0}} u+\frac{\left(\mu_{m}^{2}+\nu_{m}^{2}\right)}{2 r_{0}} u^{2}}_{\psi},
$$

where $r_{0}=\sqrt{x_{0}^{2}+y_{0}^{2}}$. If we could estimate $\psi$, then we could compute $s\left(u, x=r_{0}+\psi(u)\right)$, leading to

$$
s\left(u, r_{0}+\psi(u)\right)=a\left(y_{0}-\nu u\right) p_{c}(0) f_{m} e^{-j 2 k_{0} r(u)} .
$$

\footnotetext{
${ }^{1}$ For clearness of the exposition we considered no pointing errors of the antenna. If pointing errors are present and known, they can easily be included in the proposed approach.
}

Since $p_{c}(x)$ exhibits high resolution about $x=0$ then $s(u, x)$ becomes clustered about $x=r_{0}$, for all $u$, i.e., the range migration has been compensated.

Assume now that the received signal is immersed in white noise, $n(u, x)$, i.e.,

$$
y(u, x)=s(u, x)+n(u, x) .
$$

Define vectors

$$
\begin{aligned}
\boldsymbol{y} & \equiv\left[y_{-N}, \ldots, y_{0}, y_{N}\right]^{T} \\
\boldsymbol{s} & \equiv\left[s_{-N}, \ldots, s_{0}, s_{N}\right]^{T},
\end{aligned}
$$

where,

$$
\begin{aligned}
y_{i} & =y\left(u_{i}, r_{0}-\psi\left(u_{i}\right)\right) \\
s_{i} & =a\left(y_{m}-\nu_{m} u_{i}\right) e^{-j 2 k_{0} r\left(u_{i}\right)},
\end{aligned}
$$

with $u_{i}=u_{s} i$, where $u_{s}$ is the sampling space in the crossrange direction, $i=-N, \ldots, 0, \ldots, N$, where $2 N+1$ is the number of samples on the cross-range direction.

Let us assume that the moving target parameters $\boldsymbol{\theta}=$ $\left(y_{0}, \mu_{m}, \nu_{m}\right)$ and the reflectivity $f_{m}$ are known. In this case only the noise term in expression (6) is random. Therefore, the density of vector $\boldsymbol{y}$ conditioned to $\boldsymbol{\theta}$ and $f_{m}$ is

$$
p\left(\boldsymbol{y} \mid f_{m}, \boldsymbol{\theta}\right)=\mathcal{N}\left(\boldsymbol{m}_{\boldsymbol{y}}, \mathbf{C}_{\mathbf{y}}\right)
$$

where the mean $\boldsymbol{m}_{\boldsymbol{y}}=f_{m} \boldsymbol{a}(\boldsymbol{\theta})$ and the covariance $\boldsymbol{C}_{\boldsymbol{y}}=$ $\sigma_{n}^{2} \boldsymbol{I}$, where $\sigma_{n}^{2}$ is the noise power. Notice that $y_{i}=y_{i}(\boldsymbol{\theta})$. Therefore the density in (7) is correct only for the true $\boldsymbol{\theta}_{0}$. However, for $\boldsymbol{\theta} \approx \boldsymbol{\theta}_{0}$ the range migration is negligible and $y(\boldsymbol{\theta}) \approx y\left(\boldsymbol{\theta}_{0}\right)$. Thus, we still use (7) to derive the maximum likelihood (ML) estimator of $\boldsymbol{\theta}$. After some algebra we achieve the ML estimator of $\boldsymbol{\theta}$,

$$
\widehat{\boldsymbol{\theta}}_{M L}=\arg \max _{\boldsymbol{\theta}} \frac{\left|\sum_{i} y_{i} s_{i}(\boldsymbol{\theta})^{*}\right|}{\sum_{i}\left|s_{i}(\boldsymbol{\theta})\right|^{2}} .
$$

Assume that $a(u)=a(-u)$ and noting that $a(u)=a\left(y_{0}-\right.$ $\nu u)=a\left[\nu\left(y_{0} / \nu-u\right)\right]$, then (8) can be rewritten as a correlation

$$
\widehat{\boldsymbol{\theta}}_{M L}=\arg \max _{\boldsymbol{\theta}} \frac{\left|\sum_{i} y_{i} s_{i-i_{0}}(\boldsymbol{\theta})^{*}\right|}{\sum_{i}\left|s_{i}(\boldsymbol{\theta})\right|^{2}}
$$

with $i_{0}=\frac{y_{0}}{\nu u_{s}}$.

The proposed methodology can be summarized as follows:

1. Obtain an image focused with $\mu=0$ and $\nu=1$ (static ground parameters). The ground will be focused and the moving targets will appear defocused and at wrong positions.

2. Detect the moving targets and estimate their approximate positions using methods such as those proposed in [10] or [11]. 
3. Isolate the defocused moving targets and re-synthesize its signatures back to the $\left(u^{\prime}, x\right)$ domain as proposed in [2]. This procedure increases the signal to noise ratio, although it introduces some correlation due to the spatial windowing.

4. For each synthesized signature proceed as follows:

- Using the synthesized signature mass center, determine a rough estimate of the target coordinates $\left(\widehat{x}_{0}, \widehat{y}_{0}\right)$;

- Redefine the cross-range origin at $\widehat{y}_{0}$, that is, $u_{0}=0$ for $u^{\prime}=\widehat{y}_{0}$. Using the redefined origin leads to $\widehat{r}_{m}=$ $\widehat{x}_{0}$;

- For each $\mu, \nu$ of interest: form the observation vector $y(u)=s\left[u, r\left(u, \widehat{x}_{0}, 0, \mu, \nu\right)\right]$ and apply the matched filter described in (9), storing the coordinate $u_{m}$ where the maximum occurs and the corresponding value.

- Consider the value $u_{m}$ for the pair $(\widehat{\mu}, \widehat{\nu})$ where the maximum of the estimator has occurred: Its value is related to the error in our rough estimate of $y_{0}$. Coordinate $y_{m}$ is thus estimated as $\widehat{y}_{m}=u_{m} \widehat{\nu}+\widehat{y}_{0}$. Coordinate $x_{m}$ is estimated by $\widehat{x}_{m}=\widehat{x}_{0}-\widehat{\mu} \widehat{y}_{0}$.

To reduce drastically the length of the search interval with respect to $\mu$ one could use the methodology proposed in [12].

Using the results from the proposed strategy we have all the information needed to achieve a focused and correctly positioned image of the moving targets.

\section{SIMULATION RESULTS}

In this section we show some simulation results. Table 1 shows the simulation parameters.

We will start by considering a point target moving with range velocity $v_{r}=-7.959 \mathrm{~m} / \mathrm{s}$ (exactly 3 times the maximum velocity imposed by the used PRF), and azimuth velocity $v_{a}=8 \mathrm{~m} / \mathrm{s}$. The true azimuth coordinate of the target is $y_{m}=209$ when the platform is at the center of the target area $(u=0)$. In Fig. 3 the velocity estimation results are presented. Figure 3 plots the azimuth uncertainty estimation. Both graphics plot the results of 64 monte-carlo trials, as function of the signal-to-noise ratio. The noise are the contributions of the static ground which lie in the observation vector $y\left(u^{\prime}\right)$ formed at step 4 of the algorithm presented in previous section. The static ground was simulated as having constant intensity but phase which is uniformly distributed in a $2 \pi$ interval.

The achieved results enable both the focusing of the moving target and its repositioning with high accuray for $S N R=-9 d B$. For SNR below that value the azimuth uncertainty estimation is severely degraded and the target can no longer be correctly positioned.

Our second experiment is to use the proposed estimator with an extended moving target with homogeneous reflectivity. The velocity parameters are those of the previous
Table 1. Mission parameters used in the simulation.

\begin{tabular}{|c|c|}
\hline Parameter & Value \\
\hline Carrier frequency & $5 \mathrm{GHz}$ \\
Chirp bandwidth & $100 \mathrm{MHz}$ \\
Altitude & $12 \mathrm{Km}$ \\
Velocity & $637 \mathrm{Km} / \mathrm{h}$ \\
Look angle & $20^{\circ}$ \\
\hline
\end{tabular}

Table 2. Estimation results for three types of targets $(S N R=0 d B)$. All the targets move with range speed of $-7.959 \mathrm{~m} / \mathrm{s}$ and azimuth speed of $8 \mathrm{~m} / \mathrm{s}$. The range speed is three times the maximum imposed by the used PRF.

\begin{tabular}{|c|c|c|c|}
\hline Target type & $v_{r}$ error & $v_{a}$ error & $y_{m}$ error \\
\hline \hline point-like & $0,005 \mathrm{~m} / \mathrm{s}$ & $0,0123 \mathrm{~m} / \mathrm{s}$ & $0,45 \mathrm{~m}$ \\
\hline $7 \times 12 \mathrm{~m}$ extended & $0,3 \mathrm{~m} / \mathrm{s}$ & $4,2 \mathrm{~m} / \mathrm{s}$ & $28,3 \mathrm{~m}$ \\
\hline $\begin{array}{l}7 \times 12 \mathrm{~m} \text { extended } \\
\text { (one predominant) }\end{array}$ & $0,041 \mathrm{~m} / \mathrm{s}$ & $0,29 \mathrm{~m} / \mathrm{s}$ & $1,6 \mathrm{~m}$ \\
\hline \hline
\end{tabular}

experiment. A degradation is expected to occur because all the theory was developed for point-like targets. The estimation results are presented in Table 2. Although the range velocity estimation is good, the cross-range speed has an error of $4,2 \mathrm{~m} / \mathrm{s}$ and the azimuth position is retrieved with an error of $28 \mathrm{~m}$. The velocity estimates enable us to retrieve a focused image of the moving target, however it cannot be correctly positioned in the image.

The last experiment consists in repeating the previous simulation but with an extended moving target which exhibits a predominant scatterer (10dB above the average reflectivity). The results are expected to enhance as the predominant scatterer will provide the estimator with data which partly behaves as a point-like target. The estimation results of both the velocity and azimuth position have improved and are also presented in Table 2. For illustration purposes, the resulting ambiguity vector estimation map is sketched in Fig. 3. The achieved results enable both the focusing and the correct repositioning of the moving target image.

\section{FINAL REMARKS}

In this work we presented a novel methodology to estimate the true velocity of moving targets with range velocity which induces a doppler shift in the slow-time frequency domain beyond the maximum imposed by the pulse repetition frequency. We have also shown that it is possible to retrieve the true position where the moving target should be imaged. Using the retrieved parameters the moving target can be fo- 


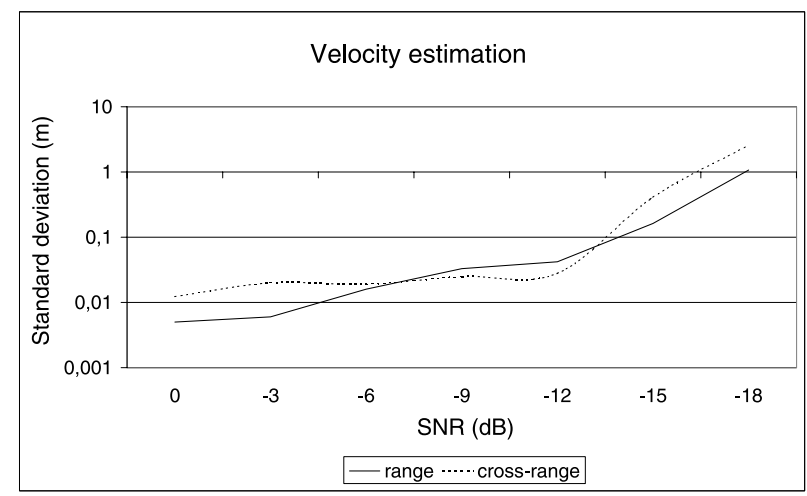

Fig. 2. Velocity estimation as function of the SNR, for 64 monte-carlo runs. The achieved results enable the focusing of the moving targets even in low SNR conditions.

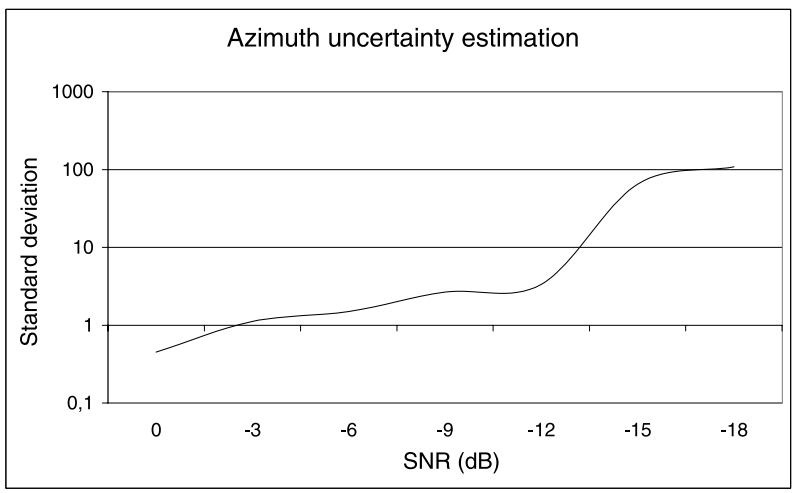

Fig. 3. Moving object position estimation as function of the SNR, for 64 monte-carlo runs.

cused and positioned correctly in the reconstructed target area. Good results where shown for point-like moving targets. The proposed technique also works well for extended targets, provided that they exhibit some predominant scatterers. When this fact does not apply, severe degradation may occur with the azimuth velocity estimation and the azimuth position estimation. More work needs to be done to model correctly extended moving targets.

\section{REFERENCES}

[1] Kazuo Ouchi, "On the multilook images of moving targets by synthetic aperture radars," IEEE Transactions on Antennas and Propagation, vol. AP-33, no. 8, pp. 823-827, August 1985.

[2] Mehrdad Soumekh, Synthetic Aperture Radar Signal Processing with MATLAB algorithms, WILEYINTERSCIENCE, 1999.

[3] Joachim H. G. Ender, "Detection and estimation of moving target signals by multi-channel sar," in Proc. of the EUSAR'96, 1996, pp. 411-417.
[4] B. Friedlander and B. Porat, "Vsar: A high resolution radar system for ocean imaging," IEEE Transactions on Aerospace and Electronic Systems, vol. 34, pp. 755-776, 1998.

[5] Genyuan Wang, Xiang-Gen Xia, Victor C. Chen, and Ralph L. Fiedler., "Detection, location and imaging of fast moving targets using multi-frequency antenna array sar (mfsar," in Proc. of the EUSAR'2000, 2000, pp. 557-560.

[6] P. Marques and J. Dias, "Optimal detection and imaging of moving objects with unknown velocity," in Proc. of the $3 r d$ European Conference on Synthetic Aperture Radar, EUSAR 2000, 2000.

[7] P. Marques and J. Dias, "Moving targets in synthetic aperture images: A bayesian approach," in Proc. of the International Conference on Image Processing, ICIP 2000, 2000.

[8] S. Barbarossa, "Detection and imaging of moving objects with synthetic aperture radar," IEE Proceedings- $F$, vol. 139, no. 1, pp. 79-88, February 1992.

[9] J. A. Legg, A. G. Bolton, and D. A. Gray, "Sar moving target detection using non-uniform pri," in Proc. of the EUSAR'96, 1996, pp. 423-426.

[10] Mehrdad Soumekh, "Reconnaissance with ultra wideband uhf synthetic aperture radar," IEEE Signal Processing Magazine, pp. 21-40, July 1995.

[11] A. Freeeman and A. Currie, "Synthetic aperture radar (sar) images of moving targets," GEC Journal of Research, vol. 5, no. 2, pp. 106-115, 1987.

[12] P. Marques and J. Dias, "Velocity estimation of fast moving targets using undersampled sar raw-data," in Proc. of the International Geoscience and Remote Sensing Symposium (to be published), IGARSS 2001, 2001.

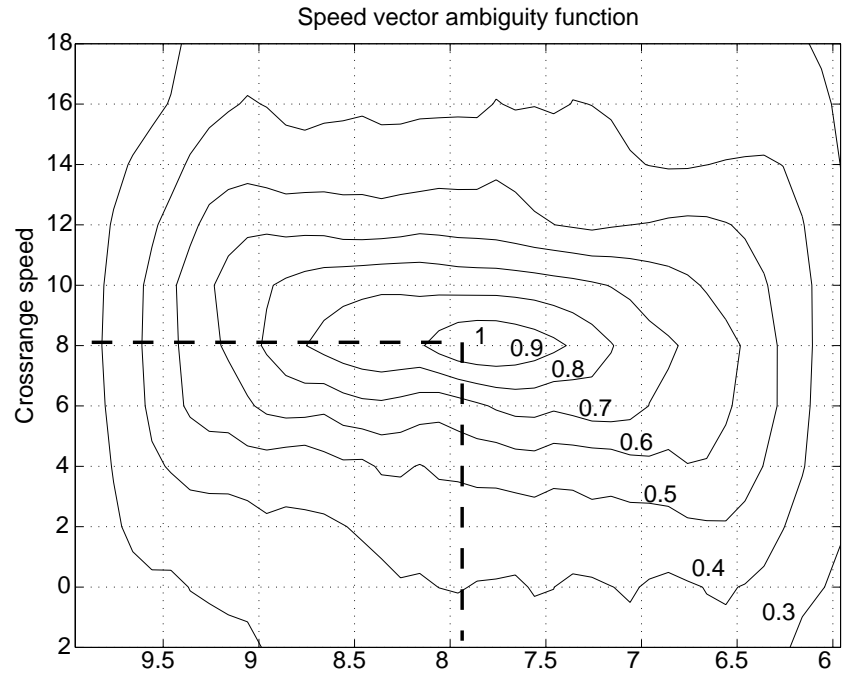

Fig. 4. Speed vector estimanange speed un uning ML estimator. 